\title{
Air rifle ammunition and its influence on wounding potential
}

\author{
W. D. F. SMITH \\ Department of Medicine, Salford Royal Hospital, Salford, England
}

\section{SUMMARY}

Two new types of air rifle pellet have been introduced; Prometheus, made from steel and nylon, and 'Sabo', made from lead alloy and plastic. Both have radio-opaque and radio-lucent components and their manufacturers claim better penetration. To establish their capabilities and the clinical implications a comparison of penetration was made between diablo, Prometheus and 'Sabo' pellets using gelatin $20 \%$ as a tissue simulant. Prometheus penetrated no further than diablo pellets but fragmented in up to $70 \%$ of cases. 'Sabo' penetrated $46 \%$ further than diablo $(p=0.001)$ and its plastic component did not penetrate at ranges greater than $0.5 \mathrm{~m}$. Prometheus penetrated steel, unlike the other pellets, and is therefore potentially more dangerous in head injury. Because of fragmentation after impact it should be remembered that the radio-lucent sleeve is likely to be lying in the wound track. 'Sabo' would appear to be the most dangerous in soft and medium density materials.

\section{INTRODUCTION}

Diablo pellets are the most widely used in the United Kingdom; they are accurate, cheap and since they have a hollow soft lead construction they expand on impact and stop quickly. Prometheus has been on the market since about 1979; it is designed for hunting and is available in $\cdot 22$ and $\cdot 177$. It is claimed to have exceptional penetration. The mushroom-shaped head is slightly subcalibre and around the stalk is a tightly fitting sleeve of nylon which provides a low friction obturation with the barrel (Cain \& Weeks, 1983; Earl, 1983). 'Sabo' was introduced more recently and is available only in -22. It is bullet-shaped to have less drag, is substantially subcalibre and is swaged into a solid pellet from lead alloy. It is loaded into the barrel using a sabot (not to be confused with 'Sabo' the trade mark) to ensure centralization and obturation (Smith, 1983). The

Correspondence: Dr W. D. F. Smith, Department of Medicine, Salford Royal Hospital, Chapel Street, Salford, M60 9EP, England 
sabot discards in flight 1-3 $\mathrm{m}$ from the muzzle and does not alter the flight of the pellet. Because drag has been reduced, velocity down-range is greater than with the other $\frac{\mathbb{\Phi}}{Q}$ ammunition and therefore penetration is greater, though no claim of increased $?$ penetration at close range is made.

\section{METHODS}

Blocks of gelatin $20 \%, 22 \times 15 \times 8 \mathrm{~cm}$, were prepared in the method described by Lewis $\&$ O'Connell (1982) and after cooling were sealed and stored in a refrigerator. Each $\vec{\circ}$ study was shot at room temperature using a $\cdot 22$ BSA Meteor air rifle which had a muzzle energy of about $7 \mathrm{ft}$. lb. The initial study with 40 rounds of each pellet was shot at $15 \mathrm{~cm} \stackrel{\omega}{\circ}$ to obtain maximum velocity and to establish if the sabot would penetrate at short range. The study was repeated at $10 \mathrm{~m}$ with 20 rounds each and a small number of rounds were also fired at denser materials such as pine blocks and 1-mm steel plate from a range of $\vec{i}$ $1 \mathrm{~m}$.

\section{RESULTS}

At $15 \mathrm{~cm}$ there was no significant difference between diablo or Prometheus pellets though Prometheus fragmented into steel and nylon components in $70 \%$ of cases. The nylon sleeve came to rest a mean of $82 \%$ of the way down the track $(65-98 \%)$ and the head remained stable. 'Sabo' penetrated $46 \%$ further than diablo which on $t$-testing was statistically significant $(p<0.001)$. The sabot penetrated the gelatin in $70 \%$ of cases to a mean distance of $1.56 \mathrm{~cm}(0-4.8 \mathrm{~cm})$ and when the range was gradually increased the sabot failed to penetrate at ranges greater than $0.5 \mathrm{~m}$.

At $10 \mathrm{~m}$ there was no significant difference between the diablo and Prometheus pellets and the Prometheus fragmented in $66 \%$ of cases. The sleeve came to rest a mean of $74 \%$ of the way down the track $(60 \cdot 4-84 \%)$. 'Sabo' penetrated a mean of $40 \%$ further than diablo, which was significant on $t$-testing $(\mathrm{p}<0.001)$. The sabot did not penetrate but was recovered intact $3-5 \mathrm{~m}$ from the muzzle.

Diablo penetrated poorly into tougher material, expanded markedly, and came to rest $1.8 \mathrm{~mm}$ below the surface of pine compared to $4 \mathrm{~mm}$ for Prometheus and $11.8 \mathrm{~mm}$ for 'Sabo'. On 1-mm steel plate diablo made a dent $1.5 \mathrm{~mm}$ deep before disintegrating. 'Sabo' also disintegrated but left a dent $2 \mathrm{~mm}$ deep. Prometheus penetrated cleanly and the head was recovered intact having separated from its sleeve. 


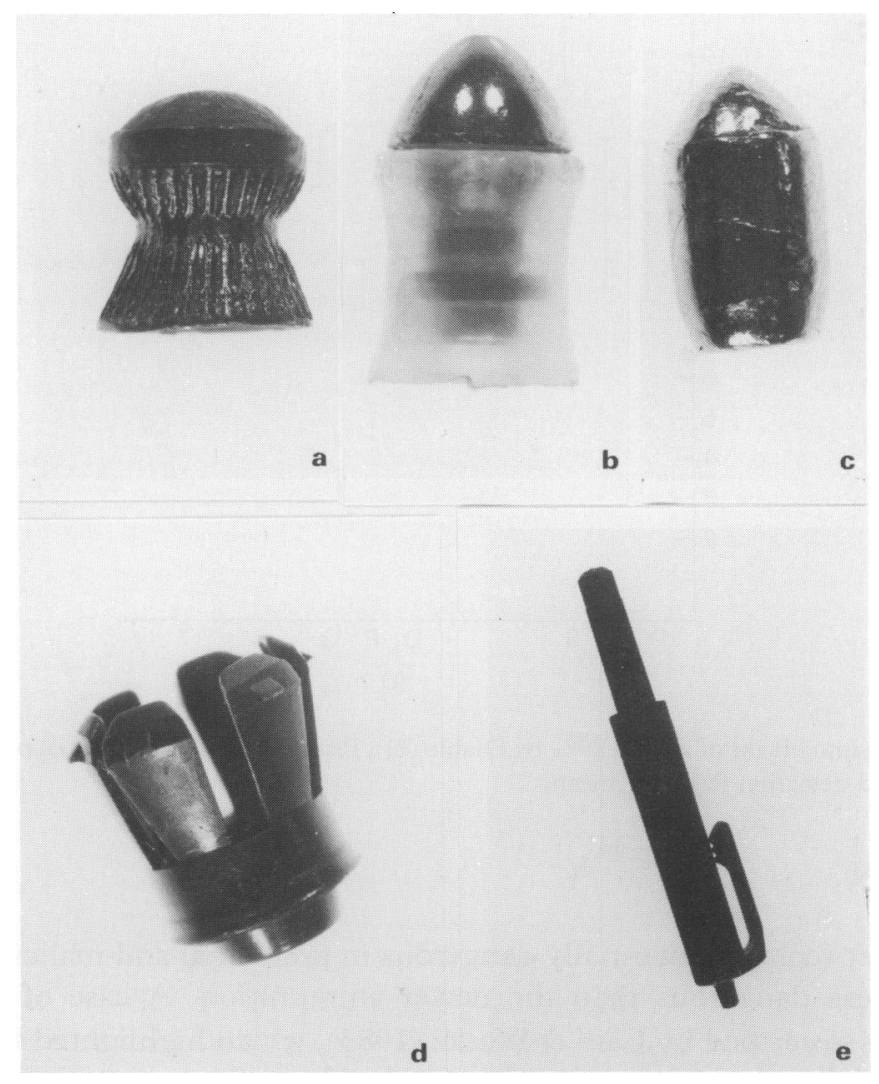

Fig. 1 Diablo (12.8 grains) (a) Prometheus (9.1 grains) (b) and 'Sabo' (13.75 grains) (c) pellets are shown with a sabot (d) and 'Sabo' dispenser (e). 


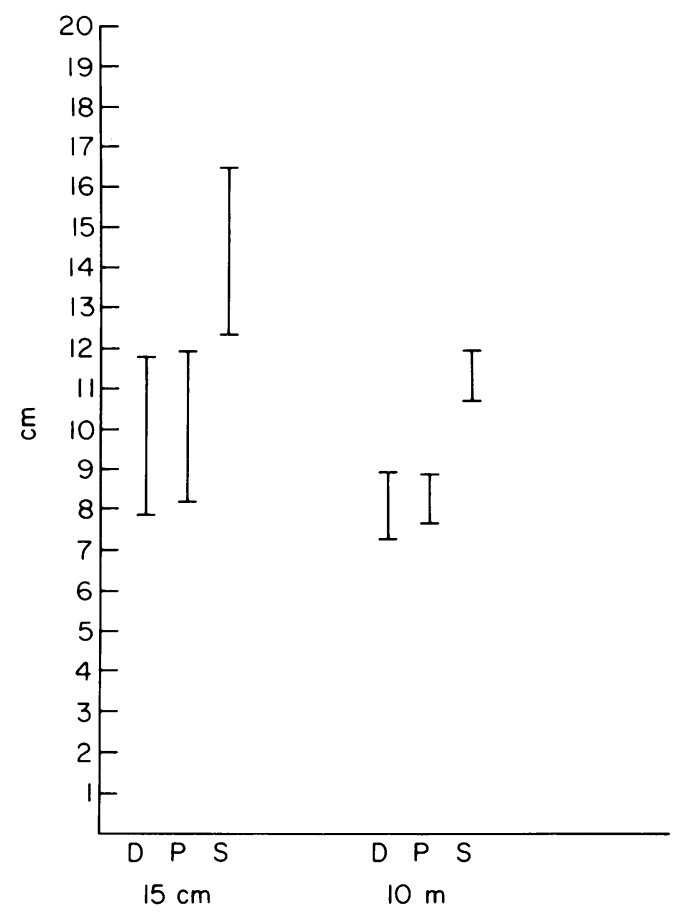

Fig. 2 Penetration into blocks of gelatin $20 \%$ by Diablo (D), Prometheus (P) and 'Sabo' pellets (S); the bas? represent 1 standard deviation from the mean.

\section{DISCUSSION}

The diablo pellet remains potentially dangerous in accidental and malicious wounding but is clearly less dangerous than the newer ammunition. A case of wounding by Prometheus was described by Cain \& Weeks (1983), which highlighted the problem of fragmentation, rather than great penetration as had been the expectation. The majority of pellets can be expected to fragment at close range and a decreasing proportion to fragment as the range increases. It is important to remember that a radio-lucent fragment is likely to be lying in the wound if exploration is being considered. Radioopacification of the sleeve would be very useful for recognition and localization.

Prometheus is clearly more dangerous on tough materials such as cranium and can be expected to penetrate thicker parts than diablo pellets. We can be thankful that it is not possible to accelerate Prometheus much above $10-12 \mathrm{ft} \mathrm{lb}$, as it separates in the barrel (D. N. Brooks, personal communication; M. H. Elliott, personal communication). N

Although 'Sabo' did not penetrate steel it penetrated soft and medium density $N$ material better and is therefore capable of causing serious penetrating head and trunk $\stackrel{N}{\mathscr{N}}$ wounds. The reason for the increased penetration probably lies in its solid swaged construction, giving it a higher sectional density (mass/diameter ${ }^{2}$ ) and therefore energy area, as the muzzle velocity is not significantly greater.

The body, not being homogenous, offers sites of varying resistance. The skin presents 
considerable resistance to penetration compared to subcutaneous tissue for missiles of this type and has not been simulated in this study. DiMaio et al. (1982) showed that 177 pellets needed a velocity of $331 \mathrm{ft} / \mathrm{sec}$ and $\cdot 22$ pellets $245 \mathrm{ft} / \mathrm{sec}$ to penetrate the skin of amputated limbs.

Clothing will offer some protection, as seen in the series described by Hutchinson (1981), where mostly limb, head and neck injury were described. The eye is a low resistance target and can be irreparably damaged by minimal penetration (Bowen \& Magauran, 1973). In contrast, the skull offers more resistance although it is not immune from penetration particularly of its thinner parts and at close range (Shaw \& Galbraith, 1977). Thus the site of injury, ammunition, weapon and the range need to be considered when assessing wounding potential. Data arising from case reports may confirm the findings and represent a case for withdrawing one or both of these new pellets.

\section{REFERENCES}

Bowen D. I. \& Magauran D. M. (1973) Ocular injuries caused by airgun pellets. An analysis of 105 cases. British Medical fournal 1, 333-7.

Cain D. \& Weeks R. F. (1983) A new danger associated with airgun pellet injuries. British Medical fournal 286, 21.

DiMaio V. J. M., Copeland A. R., Besant-Mathews P. E., Fletcher L. A. \& Jones A. (1982) Minimum velocities necessary for penetration of skin by airgun pellets and bullets. Fournal of Forensic Sciences 27, 894-8.

Hutchinson G. H. (1981) Airgun injuries in children. The Practitioner 225, 1058-60.

Lewis R. H., Clark M. A. \& O'Connell K. J. (1982) Preparation of gelatin blocks containing tissue samples for use in ballistic research. The American fournal of Forensic Medicine and Pathology 3, 181-4.

Shaw M. D. M. \& Galbraith S. (1977) Penetrating airgun injuries of the head. British fournal of Surgery 64, $221-4$.

Smith W. D. F. (1983) A new danger associated with airgun pellet injuries. British Medical fournal 286, 305.

Received 23 July 1984; accepted for publication 1 August 1984 